\title{
Evidence of slow motions by cross-correlated chemical shift modulation in deuterated and protonated proteins
}

\author{
Liliya Vugmeyster ${ }^{\mathrm{a}}$, Chiara Perazzolo ${ }^{\mathrm{a}}$, Julien Wist $^{\mathrm{a}}$, Dominique Frueh $^{\mathrm{b}} \&$ Geoffrey \\ Bodenhausen $^{\mathrm{a}, \mathrm{c}, *}$ \\ ${ }^{\mathrm{a}}$ Institut de chimie moléculaire et biologique, Ecole Polytechnique Fédérale de Lausanne, BCH, 1015 Lausanne, \\ Switzerland; ${ }^{\mathrm{b}}$ Harvard Medical School, BCMP, 240 Longwood Avenue, Boston, MA 02115, U.S.A.; ${ }^{\mathrm{c}}$ Département \\ de chimie, associé au CNRS, Ecole Normale Supérieure, 24 rue Lhomond, 75231 Paris cedex 05, France
}

Received 18 August 2003; Accepted 19 August 2003

Key words: cross-correlation rates, protein deuteration, protein side-chain dynamics

\begin{abstract}
Cross-correlated fluctuations of isotropic chemical shifts can provide evidence for slow motions in biomolecules. Slow side-chain dynamics have been investigated in ${ }^{15} \mathrm{~N}$ and ${ }^{13} \mathrm{C}$ enriched ubiquitin by monitoring the relaxation of $C^{\alpha}-C^{\beta}$ two-spin coherences (Frueh et al., 2001). This method, which had hitherto been demonstrated only for protonated ubiquitin, has now been applied to both protonated and deuterated proteins. Deuteration reduces the dipole-dipole contributions to the DD/DD cross-correlation, thus facilitating the observation of subtle effects due to cross-correlation of the fluctuations of the isotropic ${ }^{13} \mathrm{C}$ chemical shifts. The decays of double- and zero-quantum coherences are significantly slower in the deuterated protein than in the protonated sample. Slow motions are found both in loops and in secondary structure elements.
\end{abstract}

Abbreviations: NMR - nuclear magnetic resonance; DD - dipole-dipole; CSA - chemical shift anisotropy; CSM chemical shift modulation; DQ - double quantum; ZQ - zero quantum.

\section{Introduction}

Understanding internal dynamic processes in proteins is essential for elucidating various aspects of their biological function (Wand, 2001). Protein dynamics span a wide range of time-scales, and many complementary techniques are therefore necessary to characterize these processes (Palmer et al., 1996; Kay, 1998; Ishima et al., 2000; Palmer, 2001; Frueh, 2003). Recently, Frueh et al. (2001) have proposed a new approach for the investigation of slow motions on microsecond to millisecond time scales, which are much slower than the overall tumbling of the molecule, which occurs on a nanosecond timescale. The method is based on the fact that slow motions can induce correlated fluctuations of the isotropic chemical shifts of selected nuclei. An interesting feature of the method

\footnotetext{
*To whom correspondence should be addressed. E-mail:
} Geoffrey.Bodenhausen@ens.fr is that it can provide information on the existence of correlated or anticorrelated modulations. Specifically, the method has been applied to two-spin coherences involving $\mathrm{C}^{\alpha}$ and $\mathrm{C}^{\beta}$ nuclei. The difference $\Delta \Gamma^{\mathrm{AR}}$ between the relaxation rates of double (DQ) and zeroquantum (ZQ) coherences $C_{+}^{\alpha} C_{+}^{\beta}$ and $C_{+}^{\alpha} C_{-}^{\beta}$ indicates the presence of cross-correlated isotropic chemical shift modulations. The effect was found to be most pronounced for residues located in loops and near the end of $\beta$-strands and $\alpha$-helices (Frueh et al., 2001). The experiments have originally been applied to protonated ${ }^{15} \mathrm{~N},{ }^{13} \mathrm{C}$ doubly enriched ubiquitin at $\mathrm{pH} 4.5$. They have now been carried out with both protonated and deuterated ${ }^{15} \mathrm{~N},{ }^{13} \mathrm{C}$ triply labelled ubiquitin at $\mathrm{pH}$ 6.7. In deuterated proteins, deuterium-carbon dipolar cross-correlated relaxation $\left({ }^{13} \mathrm{C}-{ }^{2} \mathrm{D} /{ }^{13} \mathrm{C}-{ }^{2} \mathrm{D}\right)$ is almost negligible, so that it cannot mask the effects of cross-correlated chemical shift modulation. 
Cross-correlated chemical shift modulation (CSM/ CSM), along with cross-correlated chemical shift anisotropy (CSA/CSA) and dipole-dipole (DD/DD) mechanisms (Frueh et al., 2001) contribute to the difference $\Delta \Gamma^{\mathrm{AR}}$ between the autorelaxation rates of DQ and ZQ coherences in deuterated (Equation 1a) and protonated (Equation $1 \mathrm{~b}$ ) proteins:

$$
\begin{aligned}
& \Delta \Gamma_{\mathrm{D}}^{\mathrm{AR}}=2 \sum_{i} \Gamma_{\mathrm{C}^{\alpha} \mathrm{D}^{\mathrm{i}} / \mathrm{C}^{\beta} \mathrm{D}^{\mathrm{i}}}^{\mathrm{DD} / \mathrm{DD}}+2 \Gamma_{\mathrm{C}^{\alpha} / \mathrm{C}^{\beta}}^{\mathrm{CSA} / \mathrm{CSA}}+ \\
& 2 \Gamma_{\mathrm{C}^{\alpha} / \mathrm{C}^{\beta}}^{\mathrm{CSM} / \mathrm{CSM}} \text {, } \\
& \Delta \Gamma_{\mathrm{H}}^{\mathrm{AR}}=2 \sum_{i} \Gamma_{\mathrm{C}^{\alpha} \mathrm{H}^{\mathrm{i}} / \mathrm{C}^{\beta} \mathrm{H}^{\mathrm{i}}}^{\mathrm{DD}}+2 \Gamma_{\mathrm{C}^{\alpha} / \mathrm{C}^{\beta}}^{\mathrm{CSA} / \mathrm{CSA}}+ \\
& 2 \Gamma_{\mathrm{C}^{\alpha} / \mathrm{C}^{\beta}}^{\mathrm{CSM} / \mathrm{CSM}} \text {. }
\end{aligned}
$$

Provided the static field is not too strong, the second terms are small since the CSA tensors of $\mathrm{C}^{\alpha}$ and $\mathrm{C}^{\beta}$ nuclei are almost isotropic. In the dipolar terms $\sum_{i} \Gamma_{\mathrm{C}^{\alpha} \mathrm{D}^{\mathrm{D}} / \mathrm{C}^{\beta} \mathrm{D}^{\mathrm{i}}}^{\mathrm{DD}}$ and $\sum_{i} \Gamma_{\mathrm{C}^{\alpha} / \mathrm{H} / \mathrm{C}^{\beta} \mathrm{H}^{\mathrm{i}}}^{\mathrm{DD}}$, the summation runs over all neighbouring deuterons or protons, $\mathrm{i}=\alpha, \beta_{1}, \beta_{2}, \beta_{3}$. For a deuterated protein the dipolar contribution is reduced by a factor $\left(\gamma_{\mathrm{H}} / \gamma_{\mathrm{D}}\right)^{2}=42$ compared to a protonated protein. This facilitates the observation of the third term in Equation 1a which stems from cross-correlated modulations of isotropic chemical shifts. This rate can thus be measured with enhanced precision and accuracy.

\section{Results and discussion}

Figure 1 displays some typical decay curves of DQ and ZQ coherences $C_{+}^{\alpha} C_{+}^{\beta}$ and $C_{+}^{\alpha} C_{-}^{\beta}$. There are 60 detectable residues for the deuterated and 64 for protonated ubiquitin. The additional residues are: L8, F45, L73, and R74. Four residues (E24, N25, G47, L71) were disregarded because of signal overlap in both samples. M1 was discarded in the deuterated protein due to very low signal intensity. Residues with two carbons $\mathrm{C}^{\gamma}$ and $\mathrm{C}^{\gamma^{\prime}}$ (I3, V5, I13, V17, I23 V26, I30, I44, I61, V70) were systematically excluded from the analysis because the effective couplings $\left[\mathrm{J}\left(\mathrm{C}^{\beta} \mathrm{C}^{\gamma}\right) \pm\right.$ $\left.\mathrm{J}\left(\mathrm{C}^{\alpha} \mathrm{C}^{\gamma}\right)\right]$ and $\left[\mathrm{J}\left(\mathrm{C}^{\beta} \mathrm{C}^{\gamma^{\prime}}\right) \pm \mathrm{J}\left(\mathrm{C}^{\alpha} \mathrm{C}^{\gamma^{\prime}}\right)\right]$ lead to an approximate cosine-squared modulation of the relaxation curves. The fitting procedure closely followed our earlier work (Frueh et al., 2001). Residue Q31 in protonated ubiquitin had a relative error larger than $50 \%$ and was discarded. The fits were checked for variations in the apparent rates when the effective Jcoupling constants were varied by $\pm 5 \mathrm{~Hz}$ from the
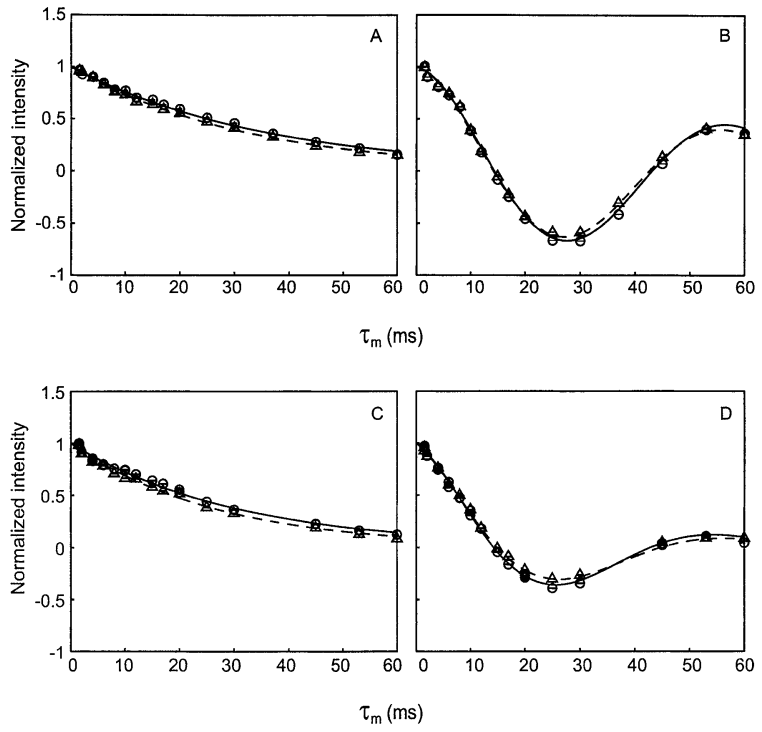

Figure 1. Examples of experimental decay curves for $\mathrm{C}^{\alpha}-\mathrm{C}^{\beta} \mathrm{ZQ}$ and DQ coherences for (A) residue $\mathrm{S} 65$ of deuterated ubiquitin which has no aliphatic $\mathrm{C}^{\gamma}$ carbons, (B) residue E16 of deuterated ubiquitin with one aliphatic $\mathrm{C}^{\gamma}$ carbon, and $(\mathrm{C})$ for residue D32 of protonated ubiquitin which has no aliphatic $\mathrm{C}^{\gamma}$ carbons, (D) residue $\mathrm{E} 16$ of protonated ubiquitin with one aliphatic $\mathrm{C}^{\gamma}$ carbon. $\mathrm{ZQ}$ signals correspond to circles with solid lines and DQ signals to triangles with dashed lines.

best-fit value. Finally, 51 and 47 residues were retained for the subsequent analysis for protonated and deuterated proteins, respectively.

The carbon-deuterium DD/DD cross-correlation rates $\left({ }^{13} \mathrm{C}_{-}^{2} \mathrm{D} /{ }^{13} \mathrm{C}-{ }^{2} \mathrm{D}\right)$ are invariant to rotations about the dihedral angle $\chi_{1}$ subtended between the two planes defined by the nuclei $\mathrm{C}^{\prime} \mathrm{C}^{\alpha} \mathrm{C}^{\beta}$ and $\mathrm{C}^{\alpha} \mathrm{C}^{\beta} \mathrm{C}^{\gamma}$. An upper limit for the sum of the rates of Eq. (1) can be calculated for a rigid protein (i.e., with an order parameter $S^{2}=1$ ) that is tumbling isotropically. A lower limit can be estimated by accounting for the effects of fast isotropic internal motions with $\mathrm{S}^{2}=$ 0.7 for $\Gamma_{\mathrm{C}^{\alpha} \mathrm{D}^{\alpha} / \mathrm{C}^{\beta} \mathrm{D}^{\alpha}}^{\mathrm{DD} / \mathrm{D}} \Gamma_{\mathrm{C}^{\alpha} \mathrm{H}^{\alpha} / \mathrm{C}^{\beta} \mathrm{H}^{\alpha}}^{\mathrm{DD} / \mathrm{D}}$ and $\mathrm{S}^{2}=0.4$ for $\Gamma_{\mathrm{C}^{\alpha} / \mathrm{DD} / \mathrm{C}^{\beta} \mathrm{D}^{\beta}}^{\mathrm{DD}}$ or $\Gamma_{\mathrm{C}^{\alpha} / \mathrm{H}^{\beta} / \mathrm{C}^{\beta} \mathrm{H}^{\beta}}^{\mathrm{DD}}$ (Lienin et al., 1998; Yang et al., 1998). In deuterated ubiquitin the ${ }^{13} \mathrm{C}_{-}{ }^{2} \mathrm{D} /{ }^{13} \mathrm{C}$ ${ }^{2} \mathrm{D}$ dipole-dipole contributions range between 0.1 and $0.3 \mathrm{~s}^{-1}$, while in protonated ubiquitin the ${ }^{13} \mathrm{C}^{-1} \mathrm{H} /{ }^{13} \mathrm{C}$ ${ }^{1} \mathrm{H}$ contributions range between 5.6 and $12.0 \mathrm{~s}^{-1}$. The CSA/CSA cross-correlation rates depend on the dihedral angle $\chi_{1}$ and hence are also sensitive to slow conformational mobility. For ubiquitin with an isotropic correlation time $\tau_{\mathrm{c}}=4.1 \mathrm{~ns}$, the CSA/CSA rates $\Gamma_{\mathrm{C}^{\alpha} / \mathrm{C}^{\beta}}^{\mathrm{CSA}}$ are expected to lie between 1.27 and $-0.63 \mathrm{~s}^{-1}$, if one assumes that the chemical shift 

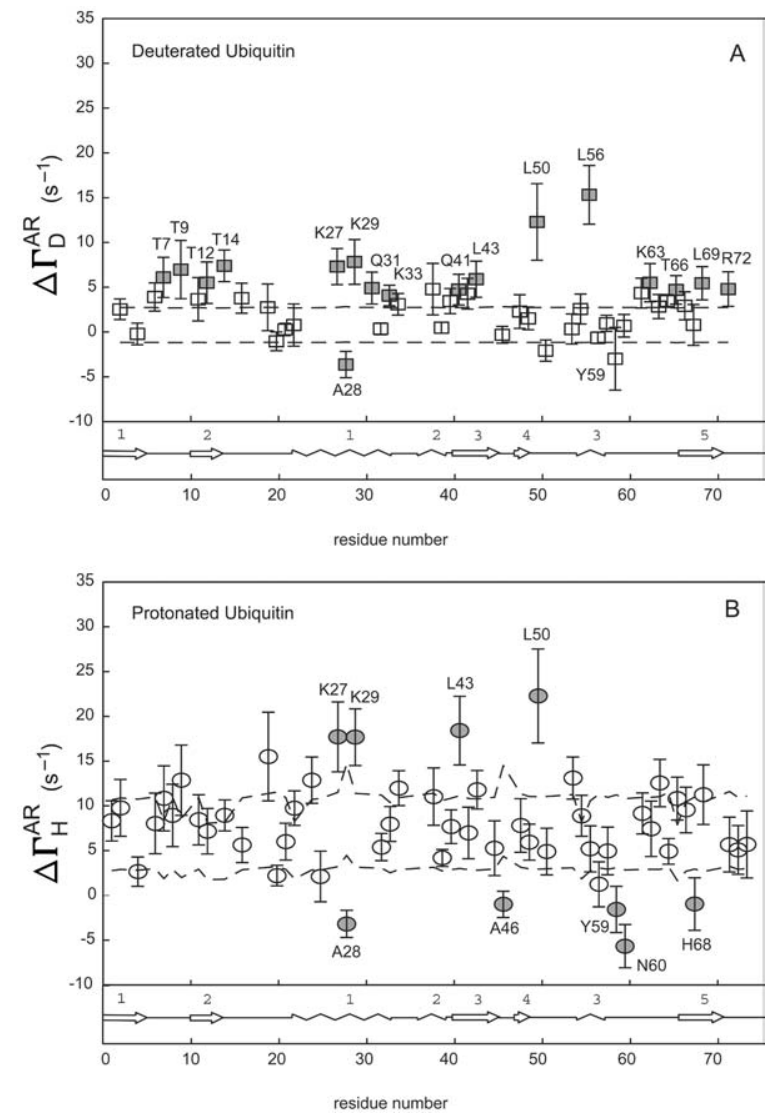

Figure 2. Experimental rates $\Delta \Gamma^{\mathrm{AR}}$ corresponding to the difference between $\mathrm{DQ}$ and $\mathrm{ZQ}$ autorelaxation rates versus residue number. (A) deuterated and (B) protonated ubiquitin. The uncertainties were obtained from nonlinear regression analysis and error propagation. The band between the two dashed lines represents the range of rates that can be explained by DD/DD and CSA/CSA cross-correlated mechanisms, the former being scaled down by a factor 42 in A, the latter being the same in both graphs. The rates that lie outside this band (filled symbols) feature cross-correlated chemical shift modulation.

tensors are axially symmetric and that the anisotropies are $\Delta \sigma_{\max }\left(\mathrm{C}_{\alpha}\right)=43 \mathrm{ppm}$ and $\Delta \sigma_{\max }\left(\mathrm{C}_{\beta}\right)=45 \mathrm{ppm}$ (Ye et al., 1993; Tjandra et al., 1997). The upper limit of $\Gamma_{\mathrm{C}^{\alpha} / \mathrm{C}^{\beta}}^{\mathrm{CSA} / \mathrm{CSA}}=1.27 \mathrm{~s}^{-1}$ is expected to be reached if the unique axes of the CSA tensors are parallel, and the lower limit of $-0.63 \mathrm{~s}^{-1}$ if they are perpendicular.

Figure 2 shows a plot of the differences of experimental ZQ and DQ relaxation rates $\Delta \Gamma^{\mathrm{AR}}$ versus residue number for deuterated (A) and protonated (B) ubiquitin. The bands between the dashed lines correspond to contributions due to the sum of (uninformative) DD/DD and CSA/CSA mechanisms. The rates that lie outside the bands cannot be explained by these two mechanisms. These rates may therefore be attributed to the CSM/CSM mechanism, and the corresponding residues must be affected by slow motions. The dashed lines in Figure 2B were calculated with a maximum rate $\Gamma_{\mathrm{C}^{\alpha} / \mathrm{C}^{\beta}}^{\mathrm{CSA} / \mathrm{CSA}}$ of $1.27 \mathrm{~s}^{-1}$ and not $0.16 \mathrm{~s}^{-1}$ as in earlier work (Frueh et al., 2001) where the CSA effects were underestimated. The DD/DD contributions are reduced by a factor $\left(\gamma_{\mathrm{H}} / \gamma_{\mathrm{D}}\right)^{2}=42$ upon deuteration. Clearly, the contributions of the CSM/CSM mechanism are easier to determine accurately in the deuterated protein, because they are less masked by dipolar effects.

As can be seen from Figure 2, 17 residues of the deuterated protein (T7, T9, T12, T14, K27, A28, K29, Q31, K33, Q41, L43, L50, L56, K63, T66, L69, R72) but only 9 residues of the protonated protein (K27, A28, K29, L43, A46, L50, Y59, N60, H68) reveal significant CSM/CSM contributions. T12, T14, Q41, L43, A46, T66, H68, L69 are located in the $\beta$-sheets, K27, A28, K29, Q31, K33 are in the first $\alpha$-helix, and T7, T9, L50, Y59, N60, K63, R72 are located in loops or at the end of secondary structure elements. Out of 9 residues that exhibit CSM/CSM contributions in the protonated sample, five (K27, A28, K29, L43, L50) also show CSM/CSM in the deuterated one. In the deuterated protein the rates of A46, Y59, N60, and $\mathrm{H} 68$ fall within the band predicted for DD/DD and CSA/CSA interactions. In the deuterated protein, twelve more residues exhibit rates that indicate CSM/CSM contributions. In the protonated protein, all of the corresponding rates, except for Q31, are within the band predicted for DD/DD and CSA/CSA interactions. Many residues in the first $\alpha$-helix exhibit CSM/CSM contributions. This observation is in agreement with evidence for slow cooperative motions in the first $\alpha$-helix (Carlomagno et al., 2000). In our previous work on protonated ubiquitin at $\mathrm{pH} 4.5$ (Frueh et al., 2001) CSM/CSM contribution could be identified only for the residues in loops or at the ends of secondary structure regions.

If we assume that CSA/CSA and CSM/CSM contributions in Equation 1 should be similar for protonated and deuterated ubiquitin, the differences in the experimental rates $\delta=\Delta \Gamma_{\mathrm{H}}^{\mathrm{AR}}-\Delta \Gamma_{\mathrm{D}}^{\mathrm{AR}}$ should give the differences of the DD/DD terms. Since the dipolar rates are almost negligible in the deuterated protein, the difference $\delta$ gives a measure of the DD/DD term $2 \sum_{i} \Gamma_{\mathrm{C}^{\alpha} \mathrm{H}^{\mathrm{i}} / \mathrm{C}^{\beta} \mathrm{H}^{\mathrm{i}}}^{\mathrm{DD}}$ in the protonated protein (Figure 3 ). The two dashed lines represent the calculated upper and lower limits for the DD/DD term. The large error bars reflect the fact that $\delta$ is obtained from a differ- 


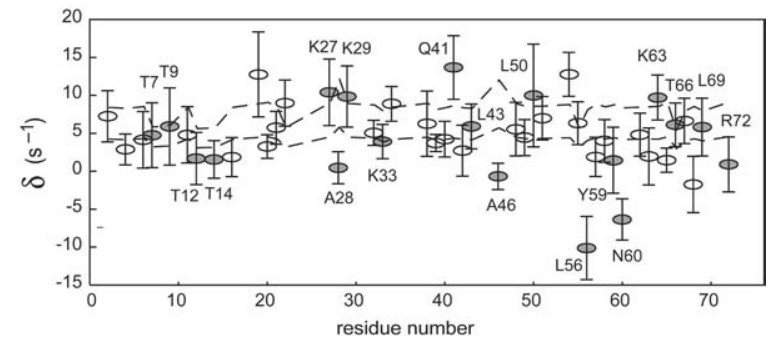

Figure 3. Differences in the experimental rates $\delta=\Delta \Gamma_{\mathrm{H}}^{\mathrm{AR}}-\Delta \Gamma_{\mathrm{D}}^{\mathrm{AR}}$ of the protonated and deuterated proteins. The band between the two dashed lines represents the expected range (see text for details). The errors are obtained from the uncertainties in $\Delta \Gamma^{\mathrm{AR}}$ by error propagation.

ence between the two rates $\Delta \Gamma^{\mathrm{AR}}$, which in turn stem from differences of DQ and ZQ autorelaxation rates. Most residues fall within the predicted band. For A28, A46, L56, N60, S65, and H68 the values are lower. This may indicate an underestimation of the influence of fast, possibly anisotropic local dynamics on the DD/DD rates. For two residues, Q41 and R54, the experimental values are higher than the predicted ones. At present, we do not have any explanation for these cases.

Depending on the details of $\mathrm{C}^{\alpha} / \mathrm{C}^{\beta} \mathrm{CSM} / \mathrm{CSM}$ interactions, they can lead to correlated or anticorrelated modulations, leading to positive or negative contributions to $\Delta \Gamma^{\mathrm{AR}}$. Only A28 shows anticorrelated modulations in both protonated and deuterated ubiquitin. In addition A46, Y59, N60 and H68 also exhibit anti-correlated CSM/CSM modulations in protonated ubiquitin. In the deuterated protein, the corresponding rates lie within the band predicted for DD/DD and CSA/CSA interactions. The results for both protonated and deuterated ubiquitin are summarised in the ribbon-diagrams of Figure 4, where side chains are shown for all residues that feature significant CSM/CSM modulations.

\section{Materials and methods}

The experimental procedure closely followed the method described for protonated ubiquitin (Frueh et al., 2001) except that for the deuterated sample proton decoupling has been replaced by WALTZ16 deuterium decoupling during the intervals where $\mathrm{C}^{\alpha}$ and $\mathrm{C}^{\beta}$ single- or multiple-quantum coherences evolve. The pulse sequence is given in 'supplementary material'. Protonated and uniformly deuterated ${ }^{15} \mathrm{~N},{ }^{13} \mathrm{C}$-enriched ubiquitin samples were obtained

\section{A Deuterated Ubiquitin}

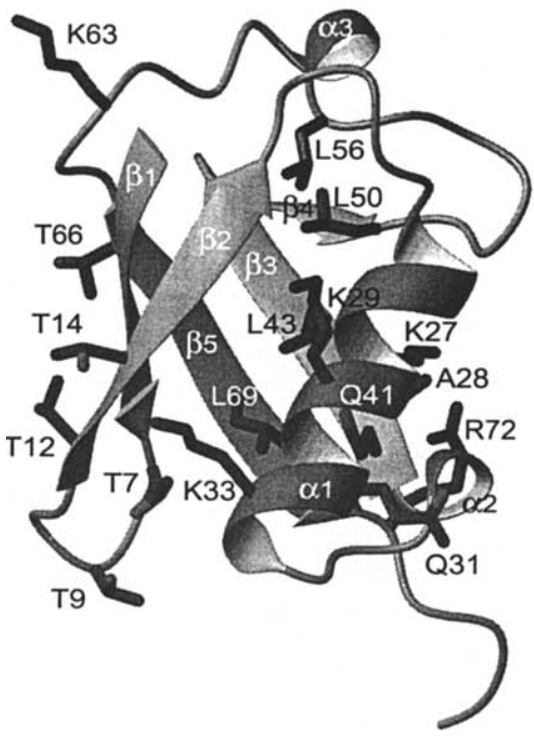

B Protonated Ubiquitin

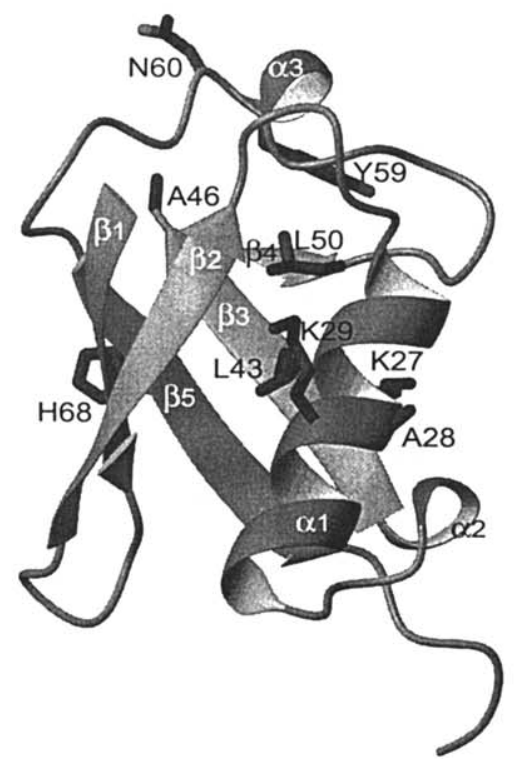

Figure 4. Ribbon representation of an X-ray structure of ubiquitin (1ubq.pdb, Vijay-Kumar et al., 1987), where side-chains are shown for residues that feature significant CSM/CSM contributions. A deuterated, B protonated ubiquitin. 
commercially (VLI). The samples were dissolved in $10 \% \mathrm{D}_{2} \mathrm{O} / 90 \% \mathrm{H}_{2} \mathrm{O}$ and the $\mathrm{pH}$ adjusted to 6.7 . The data were acquired at $303 \mathrm{~K}$ on a Bruker DMX-600 spectrometer equipped with a quadruple resonance TBI probe with triple axes gradients. Interscan delays of 1.5 and $3 \mathrm{~s}$ were used for the protonated and deuterated proteins, respectively. Each matrix consisted of 64 and 512 complex points in the $t_{1}$ and $t_{2}$ dimensions. Uncertainties of DQ and ZD rates were obtained by nonlinear regression analysis (Mosteller and Tukey, 1977). Errors in $\Delta \Gamma^{\mathrm{AR}}$ and uncertainties in the estimated DD/DD contribution for the protonated form were obtained by error propagation. A table of $\Delta \Gamma^{\mathrm{AR}}$ rates is available in 'supplementary material'.

\section{Conclusions}

Deuteration greatly reduces cross-correlated dipoledipole contributions to the decay rates of $C^{\alpha}-C^{\beta}$ zeroand double-quantum coherences, thus facilitating the observation of subtle effects due to cross-correlated modulations of the isotropic chemical shifts. The CSM/CSM rates observed in deuterated ubiquitin reveal the presence of slow motions both in loops and in secondary structure elements. The number of aminoacids that were found to feature significant CSM/CSM effects increased from 9 to 17 upon deuteration. There is only partial correlation in the residues that reveal modulations in the deuterated and protonated proteins. Further research is necessary before one could conclude that internal dynamics are affected by deuteration.

\section{Acknowledgements}

Useful discussions with Dr Jens Dittmer are acknowledged. This work has been supported by the Fonds National de la Recherche Scientifique (FNRS, Switzerland), the Commission pour la Technologie et l'Innovation (CTI, Switzerland), and the Centre National de la Recherche Scientifique (CNRS, France).

\section{References}

Carlomagno, T.M., Maurer, M., Henning, M. and Griesinger, C. (2000) J. Am. Chem. Soc., 122, 5105-5113.

Emsley, L. and Bodenhausen, G. (1990) Chem. Phys. Lett., 168, 297-303.

Emsley, L. and Bodenhausen, G. (1992) J. Magn. Reson., 97, 135148.

Frueh, D. (2003) Prog. NMR Spectrosc., 41, 305-324.

Frueh, D., Tolman, J.R., Bodenhausen, G. and Zwahlen, C. (2001) J. Am. Chem. Soc., 123, 4812-4816.

Geen, H. and Freeman, R. (1991) J. Magn. Reson., 93, 93-141.

Grzesiek, S. and Bax, A. (1993) J. Am. Chem. Soc., 115, 1259312594.

Ishima, R. and Torchia, D.A. (2000) Nat. Struct. Biol., 7, 740-743.

Kay, L.E. (1998) Nat. Struct. Biol, 5, 513-517.

Kay, L.E., Keifer, P. and Saarinen, T. (1992) J. Am. Chem. Soc., 114, 10663-10665.

Lienin, S.F., Bremi, T., Brutscher, B., Brüschweiler, R. and Ernst, R.R. (1998) J. Am. Chem. Soc., 120, 9870-9879.

Mosteller, F. and Tukey, J.W. (1977) Data Analysis and Regression: A Second Course in Statistics, 1st edn., Addison-Wesley, Reading, MA.

Palmer, A.G. (2001) Аnпи. Rev. Biophys. Biomol. Struct., 30, 129 155.

Palmer, A.G., Cavanagh J., Wright, P.E. and Rance, M. (1991) J. Magn. Reson., 93,151-170.

Palmer, A.G., Williams, J. and McDermott, A. (1996) J. Phys. Chem., 199, 13293-13310.

Shaka, A.J., Keeler, J., Frenkiel, T. and Freeman, R.J. (1983) Magn. Reson., 52, 335 .

Tjandra, N. and Bax, A. (1997) J. Am. Chem. Soc., 119, 9576-9577.

Vijay-Kumar, S., Bugg, C.E. and Cook, C.J. (1987) J. Mol. Biol., 194, 531-533.

Wand, A.J. (2001) Nat. Struct. Biol., 8, 926-931.

Yang, D., Mittermaier, A., Mok, Y.K. and Kay, L.E. (1998) J. Mol. Biol., 276, 939-954.

Ye, C., Fu. R., Hu, J., Hou, L. and Ding, S. (1993) Magn. Reson. Chem., 31, 699-704. 